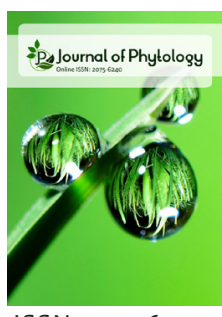

ISSN: $2075-6240$

\title{
Differential expression profiles of anthocyanidin biosynthesis gene during black rice seed development
}

\author{
Yeon Bok Kim,2, Ramaraj Sathasivam³, Soo-Un Kim Sang Un Park 3,4* \\ 'Applied Life Chemistry, School of Agricultural Biotechnology, Seoul National University, Seoul 08826, Republic of \\ Korea, ${ }^{2}$ Department of Medicinal and Industrial Crops Korea National College of Agriculture and Fisheries, Jeonju \\ 54874, Republic of Korea, '3Department of Crop Science, Chungnam National University, 99 Daehak-ro, Yuseong-gu, \\ Daejeon 34134, Republic of Korea, ${ }^{4}$ Department of Smart Agriculture Systems, Chungnam National University, 99 \\ Daehak-ro, Yuseong-gu, Daejeon 34134, Republic of Korea
}

\begin{abstract}
The black rice (Oryza sativa cv. Heugjinju) is rich in anthocyanins which is beneficial to human health. To correlate the biosynthesis of the pigments with relevant genes, the mRNA level of genes involved in anthocyanin biosynthesis was monitored by quantitative real-time polymerase chain reaction (qRT-PCR) during seed development of black rice. The mRNA level of F3'H, DFR, and ANS, key enzymes in anthocyanidin biosynthesis, peaked at 10 days after flowering. In general, the absolute level of ANS was approximately one-fold higher than F3' $\mathrm{H}, \mathrm{F} 3^{\prime} 5^{\prime} \mathrm{H}$, and DFR in 10 days after flowering. The transcript level of major seed protein gene GluA-3, taken as reference, was also at the highest on the 10 days after flowering. However, the level of CHS isogenes was highest at 15 or 20 days after flowering. The highest transcript level of the genes, except CHS, preceded the highest anthocyanidin content by 5 days. This pattern coincided with an increase of anthocyanin content between 10 and 15 days after flowering. From these findings, it is suggested that particular CHS isoforms might be responsible for the anthocyanin production in black rice.
\end{abstract}

Received: February 23, 2021 Revised: March 29, 2021 Accepted: March 30, 2021 Published: April 14, 2021

*Corresponding Author: Sang Un Park,

E-mail: supark@cnu.ac.kr

KEYWORDS: Anthocyanidin, anthocyanidin synthase, chalcone synthase, dihydroflavonol 4-reductase, flavonoid 3'-hydroxylase, flavonoid 3', 5'-hydroxylase GluA-3

\section{INTRODUCTION}

The anthocyanins belong to a widespread group of secondary metabolites collectively called flavonoids. They present in a flower petal, leaves, fruits, roots, and stem of higher plants and protect them from attracting the pollinators and animals for seed dispersal (Holton \& Cornish, 1995). The anthocyanins also have other important roles in plant ecology (Gould, 2004). For example, cyanidin 3-glucoside protects cotton leaves against the tobacco budworm (Hedin et al., 1983). The anthocyanins also have beneficial activities on human health. They possess antidiabetic, antioxidant, antimicrobial, anti-inflammatory, anti-obesity, tumor-arresting activities and it lowers the risk of cardiovascular diseases (Kong et al., 2003; Nam et al., 2005; Xia et al., 2006). Recently, efforts are being made to engineer crops to attain higher anthocyanin levels for the enhanced nutraceutical value (Shirley, 1998; Reddy et al., 2007). Black rice, an important crop in Asia, contains anthocyanin's as the major pigment components (Saito et al., 1985). The major anthocyanin in the Heugjinju variety, the most widely cultivated black rice in Korea noted for its high anthocyanin content, especially cyanidin 3-glucoside (Choi et al., 1996; Lee et al., 1998).

Anthocyanin biosynthesis involves several genes in the flavonoid biosynthetic pathway. Chalcone synthase (CHS), catalyzing the initial step in the flavonoid biosynthetic pathway, is highly regulated by the presence of multiple isozymes. Rice in particular contains a family of CHS composed of 30 members. Chalcone is converted by chalcone isomerase into naringenin, which is subsequently transformed into dihydrokaempferol (DHK) by flavanone 3-hydroxylase (F3'H), this enzyme acts on the $\mathrm{B}$ ring of DHK to result in the formation of dihydroquercetin, which is the precursor for the production of all cyanidin pigments (Fig. 1). DHK itself is the precursor of pelargonidin-type pigments (Forkmann, 1991). Additional hydroxylation on the $\mathrm{B}$ ring for blue delphinidine-based pigments requires the action of flavonol 3',5'-hydroxylase (F3'5'H) on DHK. Furthermore, the dihydroflavonols is catalyzed into leucoanthocyanidins by the enzyme dihydroflavonol 4-reductase (DFR). Dehydration of leucoanthocyanidin by the enzyme anthocyanidin synthase (ANS) is the final step for the production of anthocyanidins. 
Glycosylation of the anthocyanidins, typically at C-3' of the ring $B$ leads to the formation of various anthocyanins. The apparent color of the anthocyanidins in plant tissue is affected by various factors such as $\mathrm{pH}$ of the vacuoles, where anthocyanins are stored, complexation with metal ions, and conjugation with sugar moieties and organic acids (Strack \& Wray, 1989).

The genes involved in the anthocyanin biosynthesis have been cloned and characterized from several plants (Holton \& Cornish, 1995). However, the genes and enzymes responsible for anthocyanin biosynthesis in cereal crops have not yet been studied well (Lepiniec et al., 2006). In particular, the anthocyanin-related genes in rice were not well-characterized even though the genomic sequence of the rice has been available. The main goal of the present research is to correlate the genes putatively involved in the anthocyanin biosynthesis with the pigment accumulation in black rice. In this regard, we studied the transcription level of the related genes in a time-course manner during seed maturation.

\section{MATERIALS AND METHODS}

\section{Plant Materials}

The rice cultivar (Oryza sativa cv. Heugjinju) was grown in the month of June-October, 2006 at the field of National Crop Experiment Station, Rural Development Administration, Suwon, Korea. The seeds were harvested from 5 to 40 days after flowering.

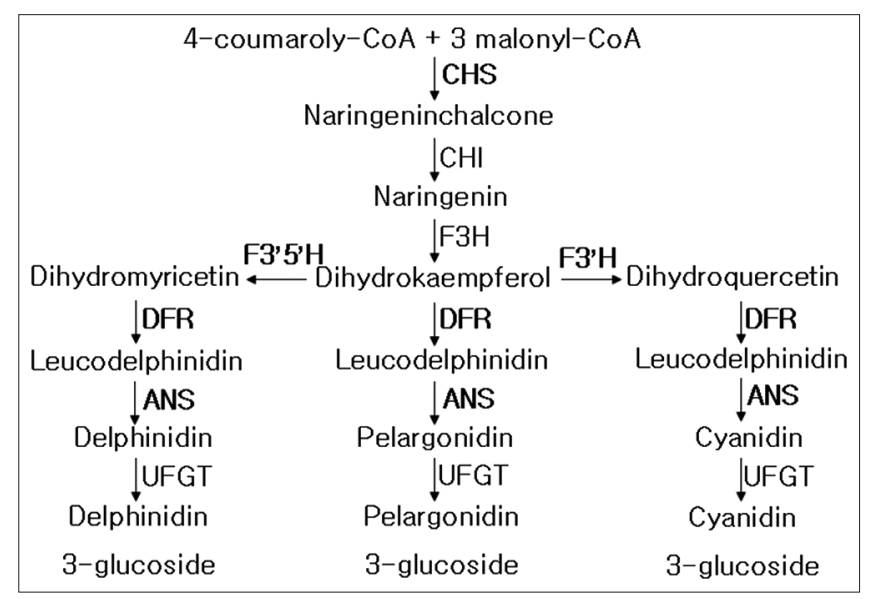

Figure 1: Simplified schematic of the anthocyanin biosynthetic pathway

\section{Total RNA Extraction and cDNA Synthesis}

Total RNA was isolated from the dehulled seeds using (Carpenter $\&$ Simon, 1998) protocol with minor modification in extraction buffer as follows: $200 \mathrm{mM}$ Tris-HCl, $300 \mathrm{mM} \mathrm{NaCl}, 20 \mathrm{mM}$ EDTA, $1 \%$ SDS, pH 8.5. Every chemical used in this study was purchased from Sigma-Aldrich St. Louis, MO, USA. Single-stranded cDNA was synthesized by the GeneRacer Kit (Invitrogen). Protocol for reverse transcriptase-polymerase chain reaction (RT-PCR) was done according to manufacture instructions (QiagenOmniscript). $1 \mu \mathrm{L}$ of cDNA was made up to $20 \mu \mathrm{L}$ volume and incubated for $60 \mathrm{~min}$ at $37^{\circ} \mathrm{C}$ and then at $95^{\circ} \mathrm{C}$ for $5 \mathrm{~min}$.

\section{Quantitative Real-time PCR and Anthocyanidin Content}

The quantitative real-time polymerase chain reaction (qRTPCR) primers were designed based on TIGR rice genome annotation (http://rice.plantbiology.msu.edu/cgi-bin/putative_ function_search.pl) (Table 1). qRT-PCR was carried out using the Rotor-Gene 2000 Real-Time Amplification System (Corbett Research, Australia) and the qRT-PCR reaction mixture was carried out according to the manufacturer protocol (Quantitect SYBR Green PCR system, Qiagen). The thermal cycling conditions were as follows; stage $1,95^{\circ} \mathrm{C}$ for $15 \mathrm{~min}$; stage $2,94^{\circ} \mathrm{C}$ for $15 \mathrm{~s}, 50^{\circ} \mathrm{C}(\mathrm{CHSl}), 53^{\circ} \mathrm{C}(\mathrm{CHS} 2$ and 3$), 52^{\circ} \mathrm{C}(\mathrm{CHS} 4), 43^{\circ} \mathrm{C}$ $\left(\mathrm{F}^{\prime} \mathrm{H}\right.$ and $\left.\mathrm{F}^{\prime} 5^{\prime} \mathrm{H}\right)$ or $57^{\circ} \mathrm{C}(\mathrm{DFR})$ for $30 \mathrm{~s}$, and stage $3,72^{\circ} \mathrm{C}$ for $30 \mathrm{~s}$. Stage 2 and 3 were repeated for 40 cycles. The reaction was run in triplicates. The standard curves for each gene, each ranging from $1 \times 103$ to $1 \times 107$ copy $/ \mu \mathrm{L}$, were prepared from PCR products of each gene as described by (Yin et al., 2001).

\section{Estimation of Anthocyanin Content}

Anthocyanin compounds were extracted after removing the rice hull and analyzed following the protocol described by Park et al., 2018.

\section{RESULTS}

\section{Transcript Level of Anthocyanidin Biosynthesis Gene in Black Rice}

There are 30 copies of CHS in the rice genome were identified (Fig. 2), among them 11 were differentially expressed. We

Table 1: Real-time PCR primers used in this study

\begin{tabular}{|c|c|c|}
\hline Genes & Forward primer $\left(5^{\prime}\right.$ to $\left.3^{\prime}\right)$ & Reverse primer $\left(5^{\prime}\right.$ to $\left.3^{\prime}\right)$ \\
\hline CHSI & CGGAGATCATCGACAAACACA & CAGCTTGAGGTCAGCGCTAGG \\
\hline CHS2 & CGTCACCACTGAGATCCCGA & GCTTGAGGTCAGCGCTTGGA \\
\hline $\mathrm{CHS} 3$ & GCATGTGCGTGTTCACGGC & ACTCCGTTGGTCGTGCAGAAC \\
\hline CHS4 & CACCCAGAGATCATTGACAAACATT & GTGCTGAAGATAAGGTGAGTGATGTC \\
\hline CHS5 & AAACCCGGCAAACATATGTCC & AGGCAATGTCTACACGAGATATTATC \\
\hline CHS6 & TCGCCAAGGACATCGCC & CGACCCCTGCCCCTAG \\
\hline CHS7 & AGCTCGAGAGGATCTATGTAGACAAG & CGATGCCGGATTTCTTACCTTG \\
\hline $\mathrm{F}^{\prime} \mathrm{H}$ & GATTCATCAACGAAAGGAAG & AGTGAATAGGTTCAGGAGCA \\
\hline $\mathrm{F}^{\prime} 5^{\prime} \mathrm{H}$ & AGCAAATCTTCTCTACGCTG & AAGTAGGTGTGCAAGTTTGG \\
\hline DFR & GTCGCGACGCCCATGGACT & GCGCTGCCGCTCCTCGATGTTGACG \\
\hline ANS & TCGTCGACATCTCCGCGTTC & ATGGGCAGCGCGAAGAACG \\
\hline GluA-3 & ATGGCAACCATCAAATTCCCTATAG & TTATGCACTCACAGATATGTCTTGGTAG \\
\hline
\end{tabular}


selected 23 CHSs longer than $1 \mathrm{~kb}$ so that all the known expressed sequences are included. Os12q07690 was excluded though it is known to be expressed because is too short to be a functional CHS. The selected genes were aligned and classified into 7 groups based on base sequence similarities and the groups were arbitrarily named CHSl through 7 (Table 2). The mismatch between the arbitrary designation and phylogenetic tree in Fig. 2 arose because the designation was based on nucleotide sequence while the tree was based on the protein sequence. It was not possible to design primers to distinguish each member in a group because of the very high base sequence homology among them. The transcripts of groups 5 through 7 were not detected by qRTPCR. The transcript levels of all CHS groups, except for CHS4, reached a maximum at 20 days after flowering. The transcript

Table 2: Arbitrary grouping of rice putative chalcone synthase in this study. The underlined are known to be expressed

\begin{tabular}{ll}
\hline Group No. & Locus Identifier \\
\hline CHS1 & Os01g12180, Os05g12210, Os05g12240, \\
& Os10g08620, Os10g08670, Os01g41834 \\
CHS2 & Os07g17010, Os07g34140, Os07g34190, \\
& Os07g34260, Os1lg32620, Os1lg32580, \\
& Osllg35930 \\
CHS3 & Os07gl1440, Os1lg32610, Os1lg32650 \\
CHS4 & Os04g01354, Os07g31770 \\
CHS5 & Os04g23940, Os10g09860 \\
CHS6 & Os07g22850, Os10g07040 \\
CHS7 & Os07g31750 \\
\hline
\end{tabular}

numbers of CHS2 and 4 in the developing seed were considerably low compared to other groups. CHS3 and CHSl levels, with maxima at 15 and 20 days after flowering respectively, were high enough to correlate with pigment accumulation, although the genes involved in the later-stage of anthocyanin biosynthesis were most active at 10 days after flowering (Fig. 3). Though the primers cannot distinguish individual CHS in a given group, only one gene is known to be expressed each in CHSl and CHS3, Os05g12210 and Os $1 \lg 32650$, respectively (Table 2). Therefore, a closer examination of the role of those genes in anthocyanin biosynthesis is necessary.

The F3'H, F3'5'H, DFR, and ANS transcripts were most abundant on the 10 days after flowering and rapidly diminished to basal level at 20 days after flowering (Fig. 4). However, the transcript level of each gene was very different from gene to gene. While the transcript level of F3'5'H was one-fold lower than F3'H and DFR, whereas ANS level was one-fold higher than F3'H and DFR (Fig. 4). To evaluate if the aforementioned anthocyanidinrelated genes follow seed development and maturation, we used GluA-3, a member of the glutelin gene family, as a reference and marker. The pattern of the transcript level of GluA-3 faithfully followed those of F3'H, F3'5'H, DFR, and ANS (Fig. 4).

\section{Anthocyanidin Pigment Content in Black Rice}

The anthocyanin content in the developing rice seed was measured to verify the temporal correlation between the

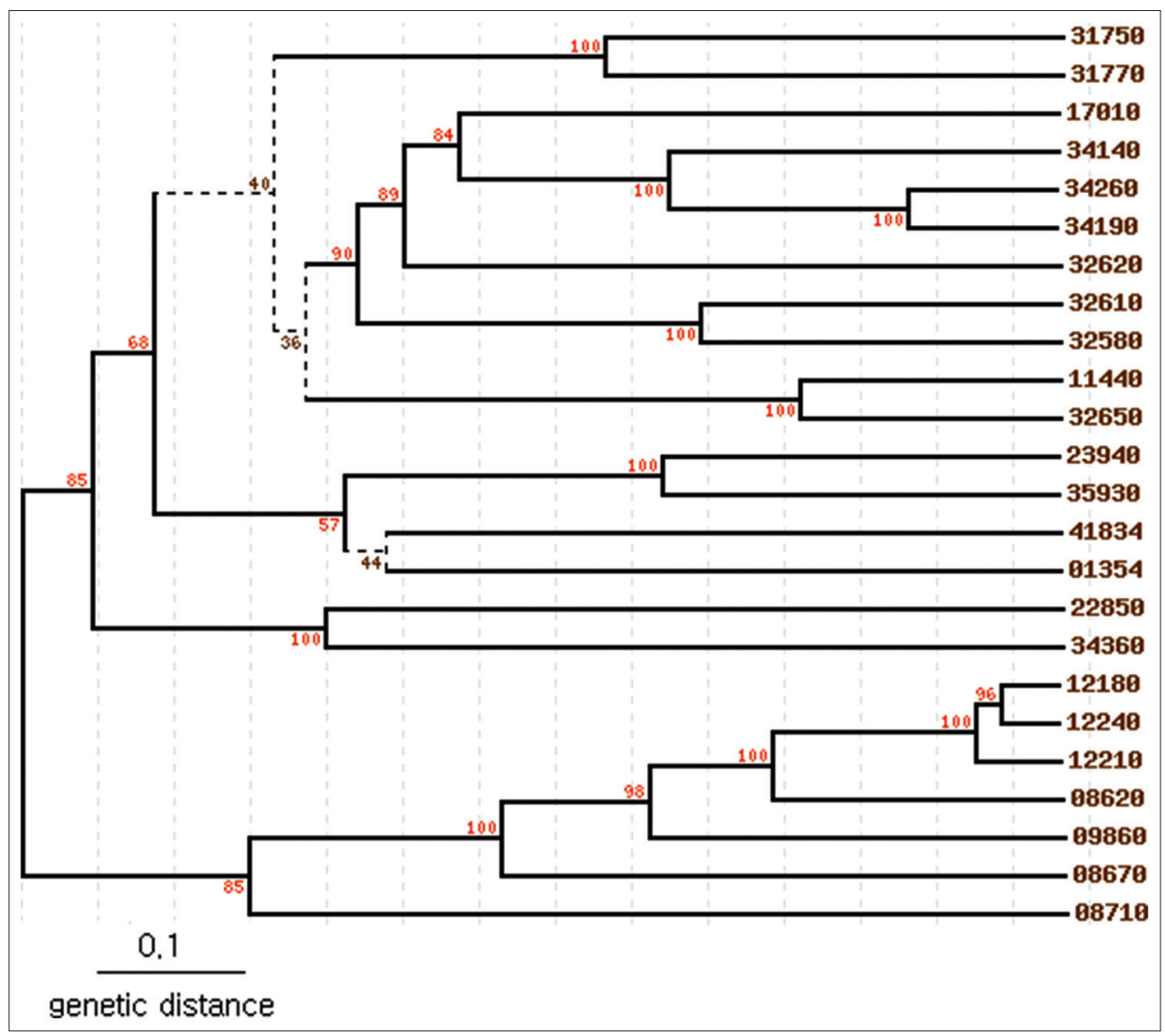

Figure 2: Phylogenetic tree of chalcone synthases in rice 


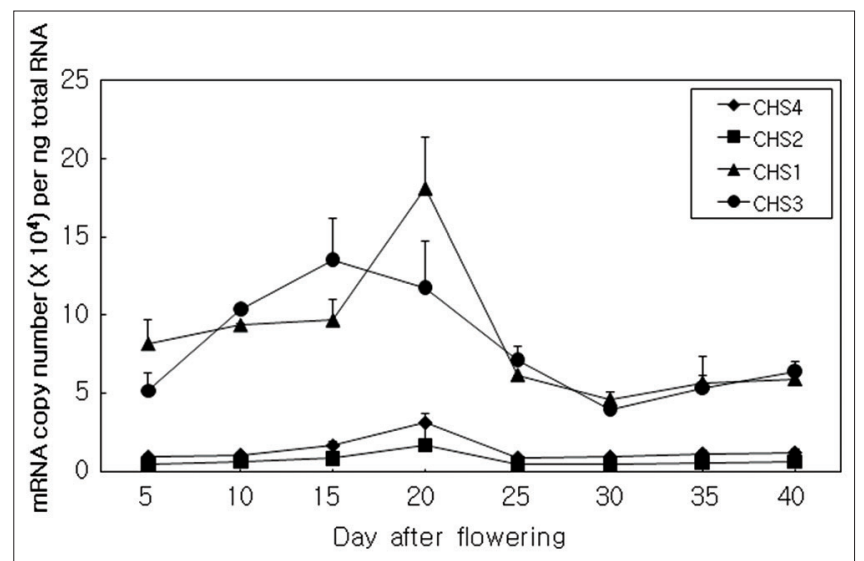

Figure 3: Quantitative real-time PCR transcript levels of chalcone synthase after flowering

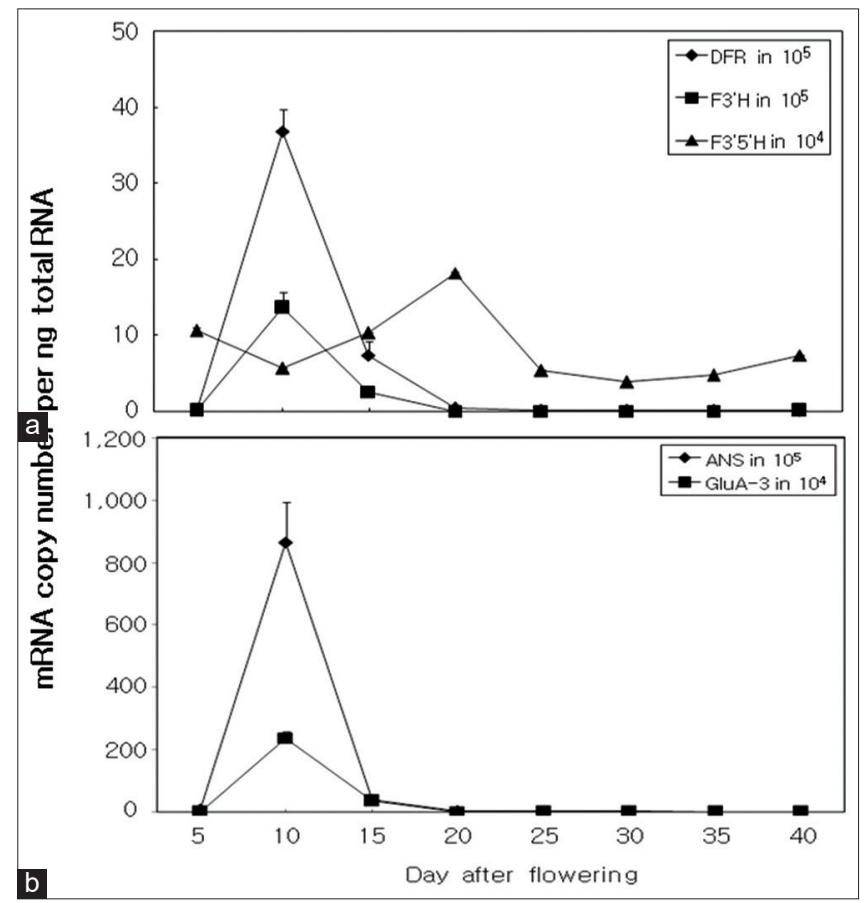

Figure 4: $\left(a\right.$ and $b$ ) Quantitative real-time PCR transcript levels of $F 3^{\prime} \mathrm{H}$, F3'5'H, DFR, ANS, and GluA-3 after flowering

transcript level and the pigment content. Five days after the pigment gene transcript levels reached maxim, that is 15 days after flowering, the pigment content reached the highest value per fresh weight, and then the content gradually decreased (Fig. 5). This timing of increase of anthocyanin content coincided with the pattern of the steady-state transcript level of the anthocyanin-related genes mentioned earlier. The anthocyanin content was reached at a maximum of $92 \mu \mathrm{g} /$ grain in the next 10 days at a rate of $1.36 \mu \mathrm{g} / \mathrm{grain} /$ day (Fig. 5).

\section{DISCUSSION}

Koes et al., 1989 identified twelve copies of CHS genes in petunia. They reported that during flower development CHS-A

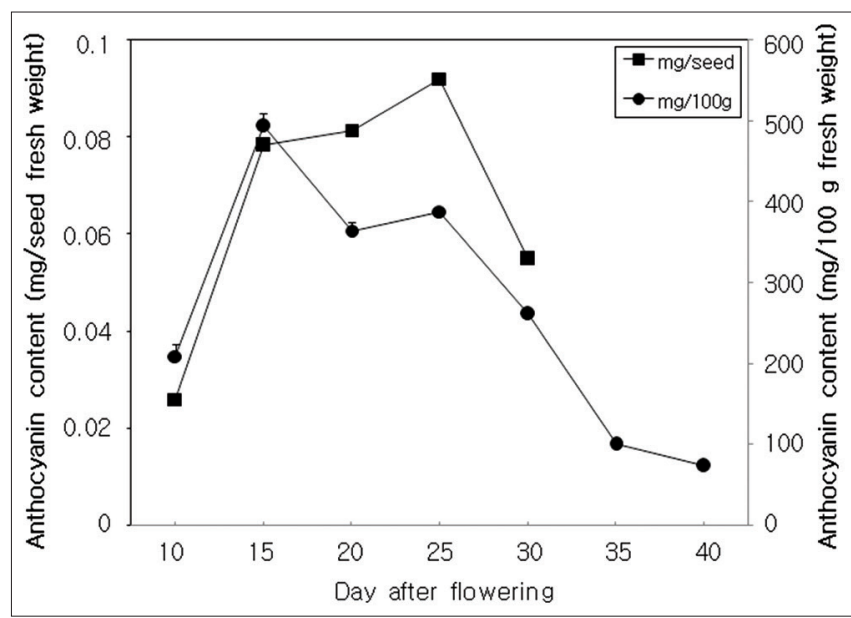

Figure 5: Anthocyanin content analysis with UV-Vis spectrophotometer after flowering

is the key CHS gene expressed, and it might encode for a major CHS protein involved in the anthocyanin biosynthesis. In addition, during flower development, CHS-J is also expressed and the expression level was about 10-fold lower than that of CHS-A (Koes et al., 1989). In black rice, the synthesis of quercetin and cyanidin (Lee et al., 1998), could be separately regulated by isozymes CHS3 and CHSl. The transcript level of CHS2, containing 5 genes known to be transcribed, and CHS4 were too low to be considered as anthocyanin-related.

F3' $\mathrm{H}$ and $\mathrm{F} 3^{\prime} 5^{\prime} \mathrm{H}$ are the two important enzymes in the flavonoid biosynthetic pathway, which leads to the production of colored anthocyanins (cytochrome P450 dependent monooxygenases and anthocyanidin synthase (Forkmann, 1991; Shimada et al., 2005). Takaiwa \& Oono, 1991 reported that the GluA-3 gene could be a useful system for studying the temporal-specific expression of seed storage protein genes. The transcript pattern of GluA-3 followed those of F3'H, F3'5'H, DFR, and ANS. This pattern supported the pattern of GluA-3 mRNA level.

The anthocyanin content increased and then the content gradually decreased. If this value was extrapolated with the fresh single grain weight (Yoshida, 1981), the anthocyanin content per grain grew most rapidly from 26 to $78 \mu \mathrm{g} /$ grain during these 5 days (10 to 15 days after flowering) at a rate of $10.5 \mu \mathrm{g} / \mathrm{grain} /$ day. This timing of increase of anthocyanin content coincided with the pattern of the steady-state transcript level of the anthocyanin-related genes mentioned earlier. The apparent decrease of the pigment level thereafter presumably reflects the rapid conversion of soluble form into polymer-bound insoluble form because the grain weight reached a plateau at 20 days after flowering.

The present results indicated that the gene transcripts for cyanidin biosynthesis in black rice were most abundant at 10 days after flowering and returned to basal level by 20 days after flowering. This temporal change of the pigment-related genes followed the same pattern as the glutelin biosynthetic gene GluA-3 transcript in rice. The behavior of those gene transcript levels was consistent with anthocyanin accumulation 
in the seed. One exception to this general pattern was CHS, whose maximal transcript level was reached 5 days later than other genes. It also retained a relatively higher level even after day 20 when other anthocyanidin genes returned to the basal level.

\section{CONCLUSION}

To correlate the biosynthesis of the pigments with relevant genes, the expression level of genes putatively involved in the anthocyanin biosynthesis was monitored by qRT-PCR during seed development of black rice. The mRNA level of F3'H, DFR, and ANS, key enzymes in anthocyanidin biosynthesis, peaked at 10 days after flowering. Among the different CHS isoforms, the CHSl and 3 showed a gradual increase in the transcript level with increasing the day after flowering. The highest level of CHSl and 3 transcripts result in the highest accumulation of anthocyanidin content at 15 or 20 days after flowering. From these results, it is concluded that the particular CHS isoforms might be responsible for the anthocyanin biosynthesis during seed development. However, in the future, further studies are necessary to determine the complete set of CHS genes in black rice and to analyze how many types of CHS isoforms involved in the anthocyanin biosynthesis during seed development

\section{ACKNOWLEDGMENTS}

This study was supported by the Ministry of Food, Agriculture, Forestry, and Fisheries through Agricultural R\&D Promotion Center, Korea.

\section{AUTHOR'S CONTRIBUTIONS}

S.U.K. and S.U.P. designed the experiments and analyzed the data. Y.B.K. and R.S. performed the experiments and analyzed the data. Y.B.K., S.U.K., and S.U.P. wrote the manuscript. All authors read and approved the final manuscript.

\section{REFERENCES}

Carpenter, C. D., Simon, A. E. (2009). Preparation of RNA. In: J. M. Martinez-Zapater, J. Salinas (Eds.), Arabidopsis Protocols. Methods in Molecular Biology ${ }^{T M}$ (Vol 82, pp 85-89) Humana Press, Springer. https://doi.org/10.1385/0-89603-391-0:85

Choi, M. -H., Paik, Y. -S., Yoon, H. -H., Hahn, T. -R. (1996). Chemical structure of the major color component from a Korean pigmented rice variety. Applied Biological Chemistry, 39(4), 304-308.

Forkmann, G. (1991). Flavonoids as flower pigments: The formation of the natural spectrum and its extension by genetic-engineering. Plant Breeding, 106, 1-26. https://doi.org/10.1111/j.1439-0523.1991. tb00474.x

Gould, K. S. (2004). Nature's Swiss army knife: The diverse protective roles of anthocyanins in leaves. Journal of Biomedicine and Biotechnology, $5,314-320$.
Hedin, P. A., Jenkins, J. N., Collum, D. H., White, W. H., \& Parrott, W. L. (1983). Multiple factors in cotton contributing to resistance to the tobacco budworm, Heliothis virescens F. In: P. A. Hedin, (Eds.), Plant resistance to insects. (Chapter 20, pp. 347-365) ACS Symposium Series. https://doi.org/10.1021/bk-1983-0208.ch020

Holton, T. A., Cornish, E. C. (1995). Genetics and biochemistry of anthocyanin biosynthesis. Plant Cell, 7(7), 1071-1083. https://dx.doi. org/10.1105\%2Ftpc.7.7.1071

Koes, R. E., Spelt, C. E., Mol, J. N. (1989). The chalcone synthase multigene family of Petunia hybrida (V30): differential, light-regulated expression during flower development and UV light induction. Plant Molecular Biology, 12, 213-225. https://doi.org/10.1007/BF00020506

Kong, J. M., Chia, L. S., Goh, N. K., Chia, T. F., Brouillard, R. (2003). Analysis and biological activities of anthocyanins. Phytochemistry, 64(5), 923933. https://doi.org/10.1016/s0031-9422(03)00438-2

Lee, H., Oh, S. -K., Choi, H. -C., Kim, S. -U. (1998). Identification of anthocyanins from pigmented rice seeds. Agricultural Chemistry and Biotechnology, 41(4), 257-260.

Lepiniec, L., Debeaujon, I., Routaboul, J. M., Baudry, A., Pourcel, L., Nesi, N., Caboche, M. (2006). Genetics and biochemistry of seed flavonoids. Annual Review Plant Biology, 57, 405-430. https://doi. org/10.1146/annurev.arplant.57.032905.105252

Nam, S. H., Choi, S. P., Kang, M. Y., Kozukue, N., Friedman, M. (2005). Antioxidative, antimutagenic, and anticarcinogenic activities of rice bran extracts in chemical and cell assays. Journal of Agricultural and Food Chemistry, 53, 816-822. https://doi.org/10.1021/jf0490293

Park, C. H.; Yeo, H. J.; Baskar, T. B.; Kim, J. K.; Park, S. U. (2018). Metabolic profiling and chemical-based antioxidant assays of green and red lettuce (Lactuca sativa). Natural Product Communications, 13(3), 315322. https://doi.org/10.1177\%2F1934578X1801300313

Reddy, A. M., Reddy, V. S., Scheffler, B. E., Wienand, U., Reddy, A. R. (2007). Novel transgenic rice overexpressing anthocyanidin synthase accumulates a mixture of flavonoids leading to an increased antioxidant potential. Metabolic Engineering, 9(1), 95-111. https:// doi.org/10.1016/j.ymben.2006.09.003

Saito, N., Abe, K., Honda, T., Timberlake, C., Bridle, P. (1985). Acylated delphinidin glucosides and flavonols from Clitoria ternatea. Phytochemistry. 24(7), 1583-1586.

Shimada, S., Inoue, Y.T., Sakuta, M. (2005). Anthocyanidin synthase in non-anthocyanin-producing Caryophyllales species. The Plant Journal, 44(6), 950-959. https://doi.org/10.1111/j.1365-313X.2005.02574.x

Shirley, B.W. (1998). Flavonoids in seeds and grains: physiological function, agronomic importance and the genetics of biosynthesis. Seed Science Research, 8(4), 415-422. https://doi.org/10.1017/ S0960258500004372

Strack, D., Wray, V. (1989). Anthocyanins. In: J. B. Harborne, (Eds.), Methods in Plant Biochemistry, Plant methods (Vol.1, pp. 325-356) Academic press, https://doi.org/10.1016/B978-0-12-461011-8.50015-9

Takaiwa, F., Oono, K. (1991). Genomic DNA sequences of two new genes for new storage protein glutelin in rice. The Japanese Journal of Genetics. 66(2), 161-171. https://doi.org/10.1266/jjg.66.161

Xia, X. D., Ling, W. H., Ma, J., Xia, M., Hou, M. J., Wang, Q., Zhu, H. L., Tang, Z. H. (2006). An anthocyanin-rich extract from black rice enhances atherosclerotic plaque stabilization in apolipoprotein e-deficient mice. The Journal of Nutrition, 136(8), 2220-2225. https://doi.org/10.1093/ jn/136.8.2220

Yin, J.L., Shackel, N.A., Zekry, A., McGuinness, P.H., Richards, C., Van Der Putten, K., McCaughan, G.W., Eris, J.M., Bishop, G.A., 2001. Realtime reverse transcriptase-polymerase chain reaction (RT-PCR) for measurement of cytokine and growth factor mRNA expression with fluorogenic probes or SYBR Green I. Immunology and Cell Biology, 79(13), 213-221. https://doi.org/10.1046/j.1440-1711.2001.01002.x

Yoshida, S. (1981). Fundamentals of rice crop science. International Rice Research Institute Institute 